\title{
Versorgung, OBELISC und die Firma Newlndex AG
}

\author{
Renato Laffranchi ${ }^{\mathrm{a}}$, Urs Stoffel ${ }^{\mathrm{b}}$ \\ ${ }^{a}$ General Manager Newlndex AG, ${ }^{b}$ Verwaltungsratspräsident Newlndex AG
}

Die NewIndex AG wurde im Jahr 2000 im Hinblick auf die künftigen tarifarischen Anforderungen unter TARMED gegründet und ist zu 100\% im Besitz der Kantonalen Ärztegesellschaften, der FMH und der Ärztekasse. Zweck von NewIndex ist die Schaffung eines gesamtschweizerischen Datenpools der schweizerischen Ärzteschaft. Unter anderem sollen damit ärzteeigene Daten für die Tarifverhandlungen mit den Versicherern zur Verfügung stehen und Abrechnungsdaten um Ärztinnen und Ärzten ein Instrument im Fall von Wirtschaftlichkeitsverfahren zur Verfügung zu stellen. Zu diesem Zwecke wurden mit der Unterstützung der NewIndex gesamtschweizerisch 10 TrustCenter geschaffen. Die Kantonalen Ärztegesellschaften haben mit dem jeweiligen TrustCenter in ihrem Kanton eine vertragliche Abmachung (Mandatsvertrag) über die Rechte und Pflichten der Datensammlung abgeschlossen.
NewIndex unterstützt mit Auswertungen, Analysen und Modellen die beiden nationalen Standesorganisationen KKA und FMH sowohl bei Taxpunktwert (TPW)-Verhandlungen wie auch bei der ausserordentlich wichtigen Revision der TARMED-Tarifstruktur. Daneben übernimmt die NewIndex aber auch ad hoc Auswertungen und Analysen für ein-

Die NewIndex AG ist zu $100 \%$ im Besitz der kantonalen Ärztegesellschaften, der FMH und der Ärztekasse.

zelne kantonale Ärztegesellschaften, für Fachgesellschaften oder Ärztegruppierungen, für verschiedene Fragestellungen im Zusammenhang mit ärztlichen Daten. Daneben macht die NewIndex aber auch Analysen und Auswertungen für nationale und kantonale Gesundheitsbehörden, wissenschaftliche In-

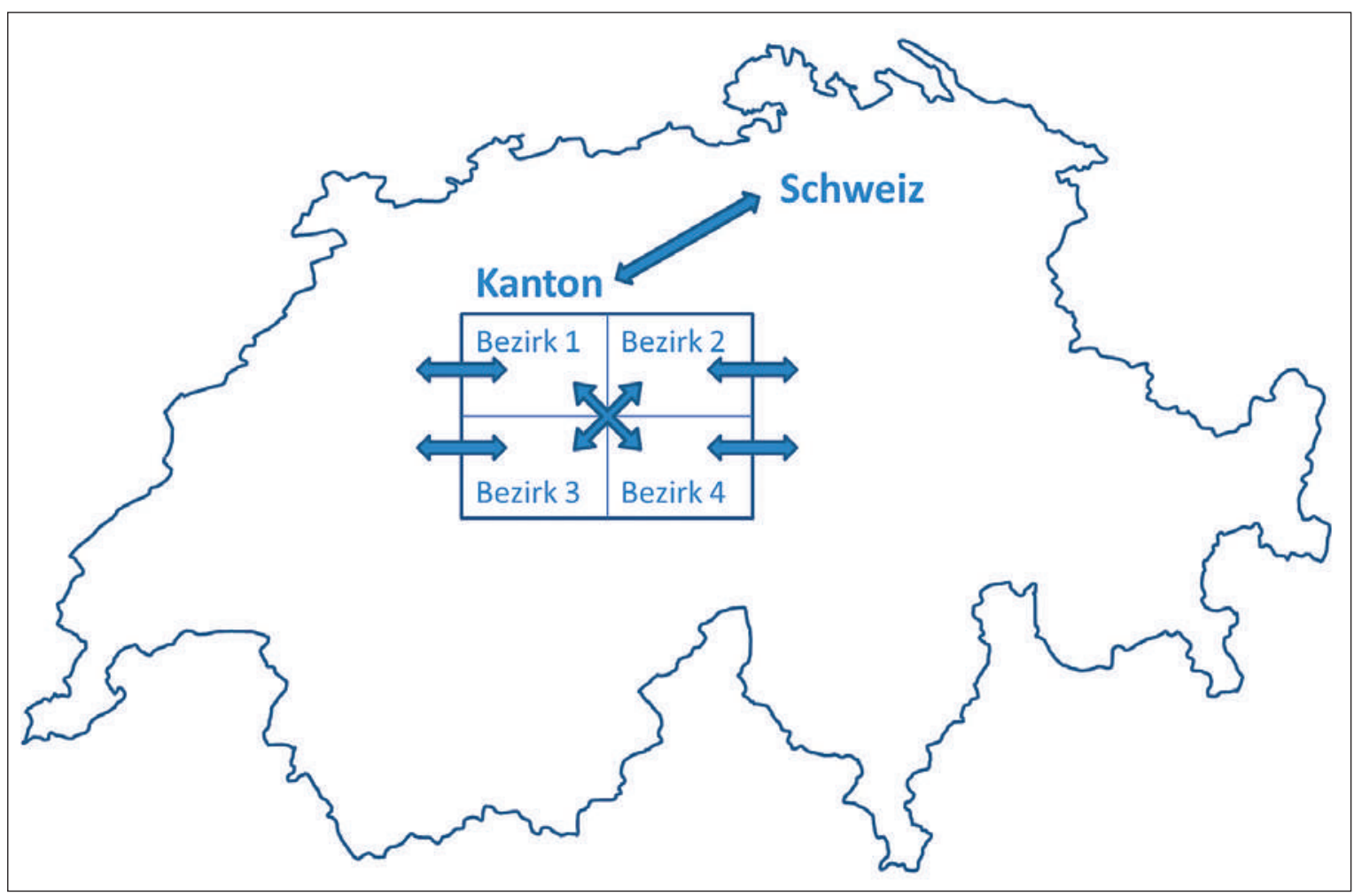

Patientenströme - wo lassen sich Patienten behandeln? 
stitute und andere im Gesundheitswesen tätige Institutionen.

Zunehmend wird die NewIndex in letzter Zeit angefragt, ob auch Fragestellungen aus der Versorgungsforschung und Monitorings für Tarifeingriffe mit dem nationalen Datenpool beantwortet werden können. Auf staatlicher Seite erhöht sich auch der Druck, Daten für Monitorings zu liefern. So werden zur Zeit entspre-

\section{Zweck von NewIndex ist die Schaffung eines gesamtschweizerischen Datenpools der schweizerischen Ärzteschaft.}

chende Klauseln in Bundes-Verordnungen eingebaut. Beim Monitoring geht es teilweise nicht nur um Volumina, sondern um eigentliche Analysen der einzelnen Sitzungen: Welche Inhalte werden während Sitzungen abgerechnet? Aufgrund solcher Sitzungsanalysen kann die Ärzteschaft besser auf ihre Bedürfnisse eingehen und entsprechend agieren und reagieren. Durch diese Art von Analysen hat die Ärzteschaft ein Alleinstellungsmerkmal, das sie unbedingt nutzen sollte.

\section{Das Projekt OBELISC}

Im Jahr 2012 hat NewIndex zur Beantwortung solcher Fragestellungen das Projekt OBELISC (OBjective Evaluation and Leadership In Scientific health data Collection) gestartet. Die Lancierung dieses Projektes war sehr erfolgreich: Bis heute machen bereits mehr als 4000 Ärzte schweizweit mit und ermöglichen es damit der NewIndex, sich nun auch im Bereich der Versorgungsforschung aktiv zu bewegen und einen für die Ärzteschaft wichtigen Beitrag zu leisten.

Im Folgenden sind zwei konkrete Fragekomplexe beschrieben, die dank OBELISC beantwortet werden können:

1. Was ist das Einzugsgebiet eines Grundversorgers oder eines Spezialisten? Woher kommen seine $\mathrm{Pa}$ tienten? Wie häufig kann ein Arzt seine Patienten aus verschiedenen Teilen seines Einzugsgebietes behandeln? Lassen sich mit der bestehenden Dichte an Grundversorgern und Spezialisten in einem Gebiet die zur optimalen Behandlung der Patienten notwendigen Richtlinien einhalten?

Antworten auf solche und ähnliche Fragen sind einerseits extrem wichtig für die Nachfolgeplanung auf Ebene Fachgesellschaft. Fachgesellschaften können damit Pläne erstellen, wie viele junge Ärzte pro Jahr und Fachspezialität ausgebildet werden müssen, um die medizinisch korrekte Versorgung der Patienten aufrechtzuerhalten. Andererseits erhalten Kantone dadurch eine zahlenbasierte Grundlage, um zu entscheiden, in welchen Gebieten wie viele Ärzte pro Fachspezialität benötigt werden.

2. Behandeln die Ärzte vor allem innerkantonale Patienten? Wie gross ist der Anteil ausserkantonaler Patienten? Was ist der Anteil der ausserkantonalen Patienten an der Kostenentwicklung im Kanton? Mit faktenbasierten Antworten auf solche Fragen können kantonale Ärztegesellschaften besser in tarifarischen Fragen argumentieren. Weiter kann dadurch der korrekte Bedarf an Ärzten besser abgeschätzt werden. Es wird Kantonen und Fachgesellschaften möglich, über die Bedeutung von Zentrumsfunktionen und konkret über Unter- oder Überversorgung Auskunft zu erhalten, um daraus notwendige Schlüsse für die Zukunft ziehen zu können.

Mit faktenbasierten Antworten können kantonale Ärztegesellschaften besser in tarifarischen Fragen argumentieren.

Wir hoffen, dass Sie als Ärztin oder Arzt durch diese Ausführungen die Wichtigkeit des Projektes OBELISC noch besser verstehen können und motiviert werden, durch die Freigabe Ihrer Daten für OBELISC einen wichtigen Beitrag für die noch bessere Nutzung des ärzteeigenen Datenpools zu leisten. Eine Teilnahme ist ganz einfach: Wenn Sie Mitglied bei einem TrustCenter sind, unterschreiben Sie die Beitrittserklärung zu OBELISC, die dieser Ausgabe der Schweizerischen Ärztezeitung beigelegt ist und schicken Sie sie an NewIndex AG, Leberngasse 19, 4600 Olten zurück.

Sie wissen nicht mehr, ob Sie bereits bei OBELISC mitmachen? Das können Sie einfach online unter www.newindex.ch/Wie-kann-ich-meine-Teilnahmeueberpruefen--103 prüfen.

Wichtig zu wissen ist auch, dass die Teilnahme bei OBELISC gratis ist und selbstverständlich alle Datenschutzauflagen erfüllt werden. 